\title{
An easier alternative to orthogonal regression for calculation of International Sensitivity Indexes
}

\author{
D A Taberner, J M K Dufty
}

\begin{abstract}
Aims-To evaluate an easier alternative method to orthogonal regression analysis for calculating International Sensitivity Indexes (ISI).

Methods-ISI for 18 reagents were estimated from reference and test reagent prothrombin times using plasma from $\mathbf{6 0}$ stabilised patients undergoing anticoagulation therapy and 20 normal subjects. ISI were also derived for 12 systems (instrument/reagent combination) using lyophilised plasma calibrants. Orthogonal regression and the easier alternative log ratio method were evaluated by comparing resultant International Normalised Ratios (INR) for 58 patients using two test sys-
\end{abstract} tems.

Results-For the reagent calibrations, the differences in the two methods for the sensitivity slopes were very small. For the system calibrations, slope differences were still of little clinical importance. Parallel observations for INR on the 58 patients confirmed that the bias introduced by easier $\log$ ratio derivation of ISI was small and of minor clinical importance, although the bias increases for high INR.

Conclusions-The easier method for ISI determination is a useful alternative to orthogonal regression analysis, particularly when computer assistance is not available, for checking for gross errors in computer computation and for use when calculating ISI from INR calibrants. (f Clin Pathol 1995;48:901-903)

Keywords: Oral anticoagulant therapy.

Prothrombin times used for monitoring oral anticoagulant therapy are now expressed as International Normalised Ratio (INR). To derive the INR, the International Sensitivity Index (ISI) of the local reagent needs to be determined. ${ }^{1}$ For assessment of the ISI of the local system (reagent/technique combination), a calibration against a reference system is required. Such calibration conventionally requires prothrombin times by local and reference systems on twenty normal subjects and 60 patients stabilised on coumarin treatment. Alternatively, a series of lyophilised plasma samples may be used to calibrate the local reagent and instrument combination sys- tem. ${ }^{2}$ The calculation of the ISI is achieved by estimating the slope (b) by orthogonal regression analysis of the logarithms of local and reference system prothrombin time results. ${ }^{1}$ Such calculation is complex. Computer programs make the calculation less tedious than using logarithm tables or a calculator, but often such programs are not locally available. We have derived a simpler method and in this study have evaluated the ISI obtained. The ISI determined by this method have been compared with those derived from orthogonal regression.

\section{Methods}

The usual method for calculating an ISI of a new prothrombin time system involves finding the slope (b) of the line of best fit (by orthogonal regression) of the log prothrombin time of the new system against the reference technique. The ISI of the new system is $b \times$ ISI of the reference technique. The easier method for determining $b$ is as follows (see appendix):

$$
\text { Slope } b=\frac{\log \text { mean reference INR }}{{ }^{*} \log \text { mean }\left(\text { local PR) }{ }^{\mathrm{ISI} \text { ref }}\right.}
$$

Eighteen calibrations of various reagents were studied. These involved parallel pro-



Figure 1 Eighteen in-house calibrations of various reagents.

* That is, log INR of local system assuming ISI is that of the reference system; PR is the prothrombin ratio. 


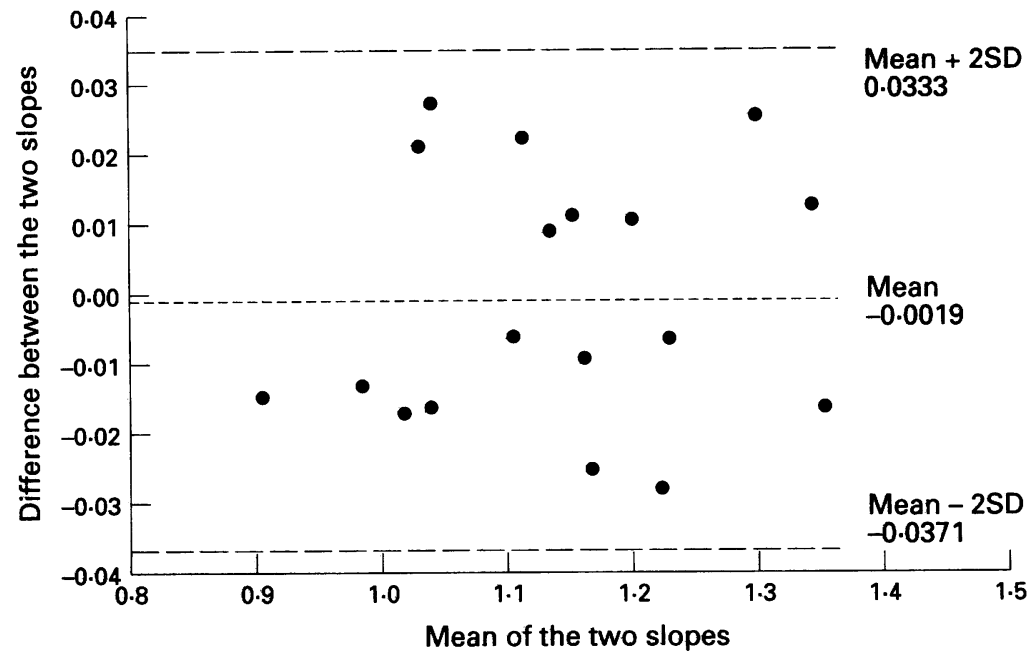

Figure 2 Bland and Altman plots of 18 calibrations using various reagents.

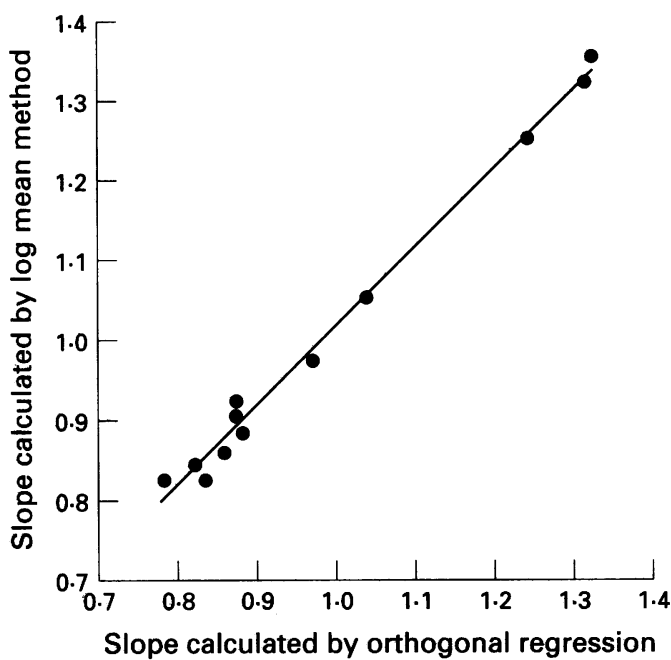

Figure 3 Twelve calibrations using lyophilised plasma samples for various machine/reagent combinations.

thrombin times using routine and reference reagents and plasma samples from 60 stabilised patients undergoing anticoagulation therapy and 20 normal subjects. The reference reagent was BCT/441 for nine, BCT/253 for six and OBT/79 for three. Slopes were calculated by the easier method and by orthogonal re-

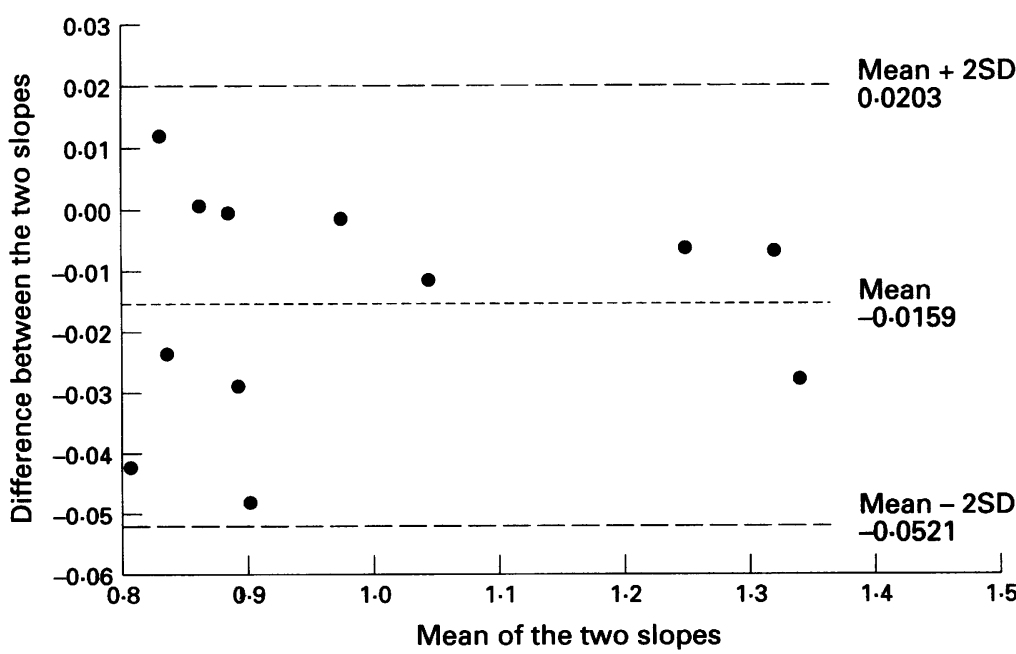

Figure 4 Bland and Altman plots of 12 calibrations using lyophilised plasma samples for various machine/reagent combinations. gression. The relation between slopes was plotted and Bland and Altman ${ }^{3}$ plots drawn.

Twelve in-house determinations of system ISI of machines using lyophilised, calibrated plasma samples ${ }^{2}$ were also examined. Again slope $b$ was calculated by the new method and by orthogonal regression. The resulting ISI were plotted and Bland and Altman plots drawn. Using ISI derived by $\log$ ratio and regression methods, the resulting INR of 58 patients receiving stable coumarin treatment were compared. This comparison was made for two of the 12 in-house determinations of system ISI.

\section{Results}

For the 18 calibrations, the mean difference between the slopes determined by the easier method and orthogonal regression was very small ( -0.0019$)$, the limits of agreement being -0.0371 to 0.0333 . For the 12 in-house machine calibrations using the 20 to 30 lyophilised plasma samples, the mean slope difference was -0.0159 with limits of agreement of -0.0521 to 0.0203 . The simple plots and Bland and Altman plots for the two types of calibrations are shown in figs $1-4$. The parallel observations on INR of 58 patient samples are shown in figs 5 and 6 by means of Bland and Altman plots. The mean INR bias for the system is small when the log mean and orthogonal derived ISI are compared.

\section{Discussion}

This new method for determining relative sensitivity slopes from thromboplastin calibration exercises, although approximate, appears to give results close to those determined by orthogonal regression. For full calibrations involving observations on 60 patients and 20 normal subjects, the slope $b$ determined by the new method is unlikely to be more than 0.0371 different from the "true" value. In INR terms this would equate to approximately 0.06 under or overestimate for an INR of $2 \cdot 17$ while for an INR of 4.23 it would give approximately 0.20 under or overestimate. When the new method is used for lyophilised plasma samples with smaller numbers of calibrants, calibrations are less close to orthogonal regression analysis. However, in clinical terms, the resultant INR is reasonably close to the estimate determined using the orthogonal regression method. This is confirmed by the parallel observations of INR on 58 patient samples, although the bias increases for high INR values.

The new method does not assess the precision of the calibration or the validity of the assumption that the slope estimate for patients and normal subjects is similar. Graphical examination of the data would permit some qualitative evaluation of such features. This method is therefore a suitable alternative for estimating ISI and may be useful where local facilities do not include orthogonal regression computer assisted analysis. It also offers a simple means of checking for gross errors in the orthogonal regression computation. In addition, it permits 




Figure 5 Bland and Altman plots of 58 INR using calibration A: MR93/10 v Ref BCT/ 441 .

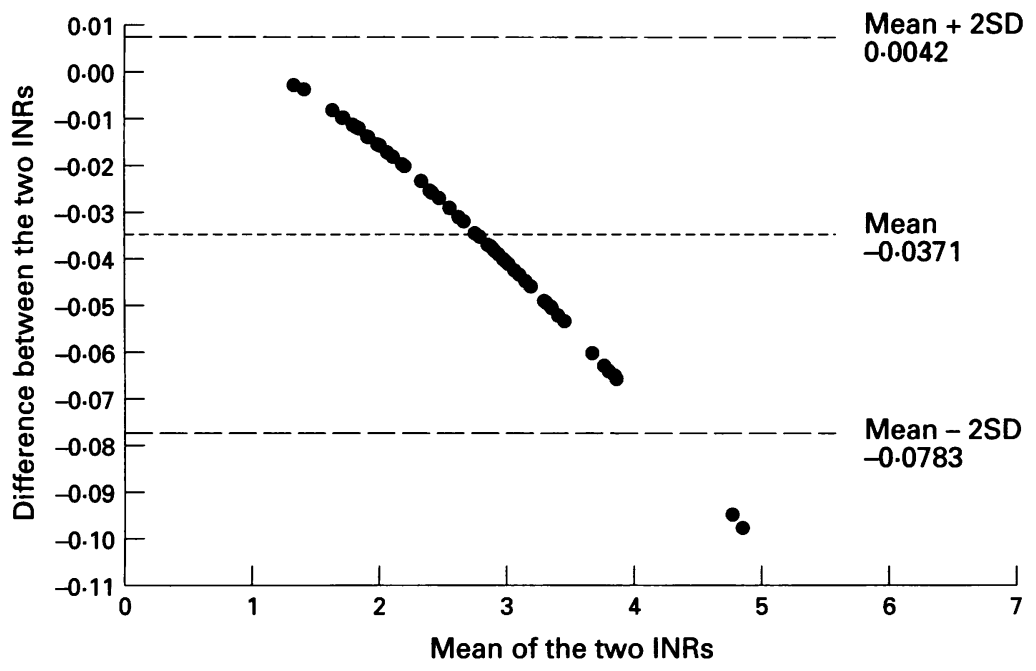

Figure 6 Bland and Altman plots of 58 INR using calibration B: Diagen v Ref BCT/ 441 .

determination of local ISI using plasma samples calibrated in terms of INR rather than using plasma calibrated in terms of prothrombin time and orthogonal regression.

1 World Health Organisation. Expert Committee on Biological Standardisation. 33rd Report. WHO Technical Report Series. Geneva: WHO, 1983:1-105.

2 Clarke K, Taberner DA, Thomson JM, Morris JA, Poller L Assessment of value of calibrated lyophilised plasmas to determine International Sensitivity Index for coagulometers. F Clin Pathol 1992;45:58-60.

3 Bland JM, Altman DG. Statistical methods for assessing agreement between two methods of clinical measurement. Lancet 1986; i:307-10.

\section{Appendix}

\section{ALGEBRAIC PROOF}

INR new system $=\left[\frac{\text { PT new system }}{\text { MNPT new system }}\right]$ ls ref $x$

where PT is the prothrombin time and MNPT is the mean normal PT.
By definition this is to be equal to the reference INR (always the "gold standard").

Thus $\left[\frac{\text { PT new system }}{\text { MNPT new system }}\right]^{\text {ISI ref } \times b}=$ INR reference

$\therefore \quad\left[\left[\frac{\text { PT new system }}{\text { MNPT new system }}\right]^{\text {IsI ref }}\right]^{b}=$ INR reference

$\therefore \quad\left[\text { local } \mathrm{PR}^{1 \mathrm{SI} \text { ref }}\right]^{b} \quad \quad=\mathrm{INR}$ reference

$\therefore \quad$ ["INR new system"] ${ }^{b} \quad=$ INR reference

if "INR new system" =[local PR $]^{\text {ISI ref }}$

Take logs of both sides of the equation

$$
\begin{aligned}
& b \times \log \text { "INR new system" = log INR reference } \\
& \therefore \quad b=\frac{\log \text { INR reference }}{\log \text { "INR new system" }}
\end{aligned}
$$
The best estimate of this is $\frac{\log \text { mean INR reference }}{\log \text { mean "INR new system" }}$

ALTERNATTVE PROOF USING A GRAPH

Plot of log times



New system

slope $(b)=\frac{v}{h}$

$=\frac{y_{2}-y_{1}}{x_{2}-x_{1}}=\frac{\log \text { time ref }-\log \text { MNPT ref }}{\log \text { time new }-\log \text { MNPT new }}$

$=\log$ (time ref/MNPT ref)

$=\frac{\log (\text { time new/MNPT new) }}{\log \text { (time }}$

$=$ ISI $\log$ (time ref/MNPT ref) where ISI is that of

$=\frac{1 S I \log \text { (time new/MNPT new) }}{\text { the reference reagent }}$

$=\frac{\text { ISI } \log (\mathrm{PR} \text { ref) }}{\text { ISI } \log (\mathrm{PR} \text { new) }}$

$=\frac{\log (\text { PR ref })^{\mathrm{ISI}}}{\log (\text { PR new })^{\mathrm{ISI}}}$

$=\frac{\log \text { INR ref }}{\log \text { "INR new" }}$

As before, the best estimate of this is $\frac{\log \text { mean INR ref }}{\log \text { mean "INR new system" }}$ 